\title{
MANEJO ANESTÉSICO PARA LA REALIZACIÓN DE BIOPSIAS TRANSBRONQUIALES GUIADAS POR ECOBRONCOSCOPIA. DESCRIPCIÓN DE 9 CASOS Y REVISIÓN DE LA LITERATURA
}

\author{
Alexo López Á., Adriana Román F., Vanesa Vilanova V., María del Carmen Corujeira R., \\ Verónica González M. e Yvan EnRIQue Sánchez S.
}

Key words: Endobronchial ultrasound, interventional bronchoscopy, intravenous anesthesia, lung cancer, trasnbronchial biospy.

\section{Resumen}

La realización de procedimientos broncoscópicos intervencionistas precisa en muchas ocasiones de la asistencia de anestesiólogos, debido al potencial compromiso de la vía aérea, a la patología asociada y a la complejidad de los procedimientos. Describimos el manejo anestésico de 9 casos de ecobroncoscopias para biopsias de ganglios en cáncer de pulmón, con anestesia intravenosa y sin la necesidad de utilizar dispositivos supraglóticos ni transglóticos, revisando a su vez la literatura al respecto.

\begin{abstract}
Anesthesists are becoming increasingly involved in the performance of interventional bronchoscopies, because of the potential risk of compromising the patency of the airway, the presence of comorbidities ant the global complexity of the procedures. We describe in this paper the anesthetic management of 9 patients scheduled for nodal transbronchial ultrasound guided biopsy with intravenous agents and without the need of supraglotic or transglotic devices.
\end{abstract}

\section{INTRODUCCIÓN}

La asistencia durante actos no estrictamente quirúrgicos implica cada vez más a especialistas en Anestesiología. A pesar de que la mayoría de las fibrobroncoscopias se realizan de forma rutinaria sin la concurrencia de anestesiólogos, ciertos procedimientos intervencionistas (biopsias transbronquiales guiadas por ecografía, resección de lesiones intraluminales, crioterapia sobre lesiones obstructivas) pueden hacer necesaria nuestra participación debido a la duración y complejidad de las intervenciones o a problemas particulares de cada paciente. Las ecobroncoscopias incluyen una serie de procedimientos novedosos y en auge, que tienen unas implicaciones anestésicas propias, entre otras, el hecho de compartir la vía aérea con el propio intervencionismo, la posibilidad de realizarse fuera del entorno del quirófano, y la elevada incidencia preoperatoria de problemas respiratorios específicos (hemoptisis, obstrucciones intrínsecas y extrínsecas de la vía aérea, patologías respiratorias crónicas). A continuación describimos el manejo anestésico de una serie de 9 pacientes en nuestro

Servicio de Anestesiología, Reanimación y Tratamiento del Dolor, Hospital Meixoeiro de Vigo (Complejo Hospitalario Universitario de Vigo). España. 
centro y su evolución en el postoperatorio inmediato.

\section{CASOS CLÍNICOS}

Recogimos de forma retrospectiva los datos sobre el manejo de 9 pacientes sometidos a ecobroncoscopias para la estadificación y estudio de enfermedades neoplásicas, estudio de adenopatías sospechosas o estudio de enfermedades pulmonares intersticiales mediante la realización de biopsias transbronquiales guiadas por ecografía endo- bronquial, y que requirieron anestesia durante su realización. Todos los casos se realizaron en un quirófano de nuestro centro por parte del mismo neumólogo y diferentes anestesiólogos. Se trata de una serie (Tabla 1) que incluye 4 pacientes considerados ASA IV, 2 pacientes ASA III, y 3 pacientes ASA II; 7 de ellos fueron hombres y 2 mujeres, y la media de edad fue de 59 años. Las indicaciones de este procedimiento fueron el estudio de lesiones sospechosas de neoplasia en 8 de los casos, mientras que en uno de ellos fue el estudio de una enfermedad pulmonar intersticial. En todas las ocasiones se realizó el procedimiento sin necesidad de usar

Tabla 1. Características clínicas de los pacientes, agentes anestésicos utilizados, indicación, procedimiento, manejo de la vía aérea y estancia en la sala de despertar

\begin{tabular}{|c|c|c|c|c|c|c|c|}
\hline Edad & ASA & Patología asociada & Indicación & Procedimiento & Agentes IV & ML/IOT & $\begin{array}{r}\text { Tiempo } \\
\text { alta }(\mathrm{H})\end{array}$ \\
\hline 62 & 4 & $\begin{array}{l}\text { Disfunción sistólica } \\
\text { no isquémica, insufi- } \\
\text { ciencia renal }\end{array}$ & $\begin{array}{l}\text { E s t u d i o } \\
\text { EPID }\end{array}$ & BTB ecoguiada & $\begin{array}{l}\text { Midazolam, } \\
\text { propofol }\end{array}$ & No & 3,5 \\
\hline 51 & 3 & $\begin{array}{l}\text { Obesidad mórbida } \\
\text { (IMC 53), trastorno } \\
\text { delirante }\end{array}$ & $\begin{array}{l}\text { Sospecha/ } \\
\text { estudio neo- } \\
\text { plasia }\end{array}$ & BTB ecoguiada & $\begin{array}{l}\text { Midazolam, } \\
\text { propofol }\end{array}$ & No & 2 \\
\hline 25 & 2 & $\begin{array}{l}\text { Trastorno pulmonar } \\
\text { restrictivo, FA }\end{array}$ & $\begin{array}{l}\text { Sospecha/ } \\
\text { estudio neo- } \\
\text { plasia }\end{array}$ & BTB ecoguiada & $\begin{array}{l}\text { Midazolam, } \\
\text { propofol }\end{array}$ & No & 2 \\
\hline 48 & 2 & $\begin{array}{l}\text { TEP bilateral, esqui- } \\
\text { zofrenia, esplenec- } \\
\text { tomía }\end{array}$ & $\begin{array}{l}\text { Sospecha/ } \\
\text { estudio neo- } \\
\text { plasia }\end{array}$ & BTB ecoguiada & $\begin{array}{l}\text { Midazolam, } \\
\text { prop of o } 1, \\
\text { fentanilo }\end{array}$ & No & 2 \\
\hline 77 & 3 & Silicosis, ACV, FA & $\begin{array}{l}\text { Sospecha/ } \\
\text { estudio neo- } \\
\text { plasia }\end{array}$ & BTB ecoguiada & $\begin{array}{l}\text { Midazolam, } \\
\text { propofol }\end{array}$ & No & 2,5 \\
\hline 54 & 4 & $\begin{array}{l}\text { Cardiopatía isqué- } \\
\text { mica, disfunción sis- } \\
\text { tólica, hipertensión } \\
\text { pulmonar }\end{array}$ & $\begin{array}{l}\text { Sospecha/ } \\
\text { estudio neo- } \\
\text { plasia }\end{array}$ & BTB ecoguiada & Propofol & No & 1,5 \\
\hline 76 & 4 & $\begin{array}{l}\text { Cardiopatía isquémi- } \\
\text { ca, enfisema pulmo- } \\
\text { nar, panhipopituita- } \\
\text { rismo }\end{array}$ & $\begin{array}{l}\text { Sospecha/ } \\
\text { estudio neo- } \\
\text { plasia }\end{array}$ & BTB ecoguiada & $\begin{array}{l}\text { Midazolam, } \\
\text { fentanilo }\end{array}$ & No & 2 \\
\hline 58 & 4 & $\begin{array}{l}\text { Síndrome Eaton Lam- } \\
\text { bert }\end{array}$ & $\begin{array}{l}\text { Sospecha/ } \\
\text { estudio neo- } \\
\text { plasia }\end{array}$ & BTB ecoguiada & $\begin{array}{l}\text { Midazolam, } \\
\text { propofol }\end{array}$ & No & 1 \\
\hline 52 & 4 & $\begin{array}{l}\text { Cardiopatía isqué- } \\
\text { mica }\end{array}$ & $\begin{array}{l}\text { Sospecha/ } \\
\text { estudio neo- } \\
\text { plasia }\end{array}$ & BTB ecoguiada & Propofol & No & 2 \\
\hline
\end{tabular}

EPID: enfermedad pulmonar intersticial difusa; BTB: biopsia transbronquial; ML: mascarilla laríngea; IOT: intubación orotraqueal; IMC: índice de masa corporal; ACV: accidente vascular cerebral; TEP: tromboembolismo pulmonar; FA: fibrilación auricular. 
dispositivos, ni supraglóticos ni transglóticos, para asegurar la vía aérea. La monitorización consistió en todos los casos en electrocardiograma de una derivación continuo, tensión arterial no invasiva y saturación periférica. En todos ellos se utilizaron los mismos agentes anestésicos intravenosos: midazolam, fentanilo y propofol, solos o combinados. La asociación más utilizada fue midazolam y propofol ( 5 casos); seguido en frecuencia de propofol solo (2 casos); midazolam, propofol y fentanilo (1 caso); y midazolam con fentanilo ( 1 caso). La media y la moda del tiempo de alta desde la Sala de Despertar fue de 2 horas (valores máximo y mínimo de 3,5 horas y 1 hora). La forma de administrarlos quedó a criterio del anestesiólogo responsable, de manera que en 6 casos la administración se realizó mediante bolos, que fueron de midazolam 0,33-0,66 mg/ $\mathrm{Kg}$, propofol $0,5^{-1} \mathrm{mg} / \mathrm{Kg}$ (en un caso, de mayor duración fueron necesarios hasta $10 \mathrm{mg} / \mathrm{Kg}$ en bolos fraccionados) y fentanilo entre 2 y 4 microgramos/ $\mathrm{Kg}$. Hubo 5 casos en los que se utilizó perfusión de propofol mediante sistema target controlled infusion (TCI) con una concentración objetivo entre 2 y $3 \mathrm{microgr} / \mathrm{mL}$. No se registró ninguna complicación relevante durante el postoperatorio, ni hipoxemias, ni desaturaciones, ni inestabilidad hemodinámica significativa; en un caso apareció una desaturación hasta $85 \%$ de $\mathrm{SaO}_{2}$ periférica que se resolvió con tracción mandibular y extensión cervical.

\section{DISCUSIÓN}

La realización de biopsias transbronquiales, guiada por ecografía endobronquial, constituye una técnica relativamente novedosa y con unas particularidades propias en cuanto al manejo anestésico. Es una de las técnicas invasivas no quirúrgicas, junto con la ultrasonografía endoscópica digestiva, disponibles para el estadiaje de enfermedades neoplásicas ${ }^{1}$, y cada una de ellas permite acceder a diferentes grupos de ganglios, como se muestra en la Figura 1. Consiste esencialmente en el acceso a estructuras adyacentes a la pared traqueobronquial, así como parénquima pulmonar, para su análisis histológico o microbiológico guiados por una imagen en tiempo real mediante ultrasonidos; existen dos tipos, la de visión radial, mediante transductores rotatorios miniaturizados que emiten ultrasonidos perpendiculares al eje del endoscopio, diseñados con un balón inflable en la punta del endoscopio para conseguir un contacto completo con la pared bronquial; el segundo tipo sería el lineal, que permitiría además diferenciar mediante doppler color pulsado las estructuras vasculares, como ocu-

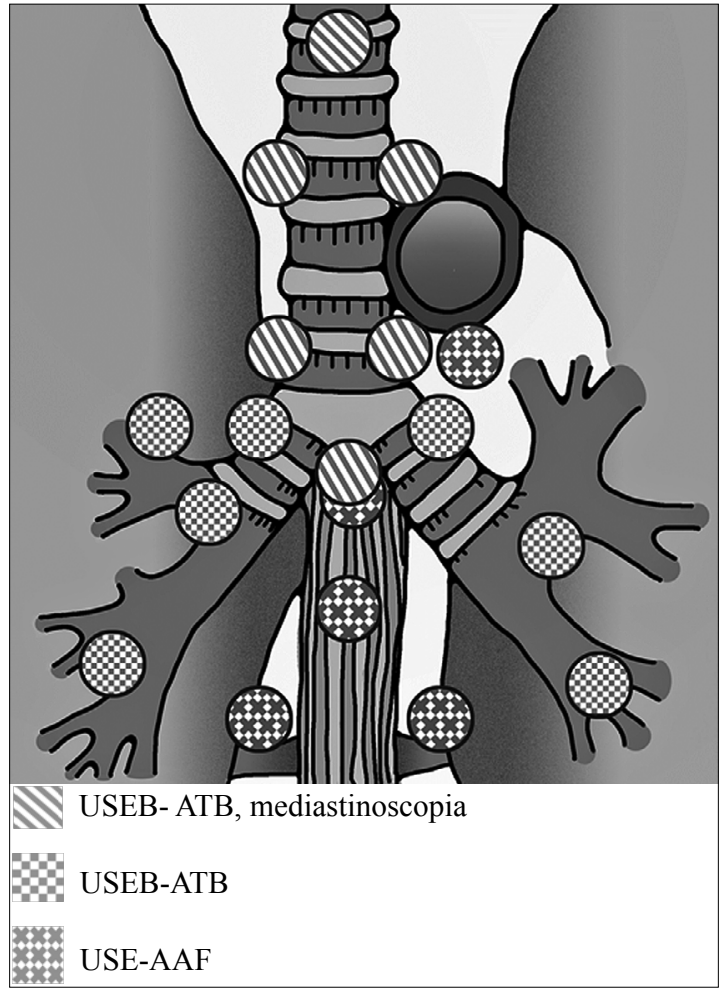

Figura 1. Técnicas disponibles para acceder a diferentes grupos de ganglios, interpretación: USEB- ATBultrasonografía endobronquial-aspiración transbronquial. USE-AAF- ultrasonografía endoscópica-aspiración con aguja fina.

rrió en nuestra serie. Para la realización de estas técnicas se requiere una inmovilidad casi completa del paciente con el fin de garantizar que se obtiene un rendimiento adecuado del procedimiento dada la relevancia posterior para definir su pronóstico. Además requiere que tanto el neumólogo como el anestesiólogo compartan el manejo de la vía aérea, el primero como forma de acceso al lugar donde se realiza el intervencionismo, y el segundo para intentar garantizar la permeabilidad de la vía aérea, especialmente en el caso de que se opte por prescindir de dispositivos, como ocurrió en todos nuestros casos. Se trata de pacientes con un riesgo perioperatorio significativo por tener mayor asociación con enfermedades respiratorias (probablemente por la relación de cáncer, patologías respiratorias y tabaquismo) y riesgo de complicaciones con la vía aérea (hemoptisis y obstrucciones mecánicas).

Aunque existe poca bibliografía respecto al manejo por anestesiólogos durante estas técnicas, en las referencias que hemos encontrado se defiende 
el uso de anestesia general con los mismos agentes que nosotros utilizamos pero preconizando como técnica de elección el uso de diferentes dispositivos para garantizar la permeabilidad de la vía aérea conjuntamente y el uso de relajantes neuromusculares, utilizándose en la mayoría de los casos, la mascarilla laríngea (al menos del número 4), y en algunos casos la intubación orotraqueal (con un diámetro de 8,5-9 para garantizar que se mantiene la ventilación durante la introducción del broncoscopio). La ventaja de utilizar la mascarilla laríngea frente a la intubación orotraqueal (Figuras 2 y 3 ) es que algunos de los ganglios a los que se pretende acceder durante el procedimiento quedarían inaccesibles debido a una deficiente alineación con los haces de ultrasonidos, de manera que el tubo endotraqueal impediría acceder a ellos de forma adecuada a través de una punción transtraqueal ${ }^{2,3,4}$.

Parece que la utilización de anestésicos intravenosos tendría varias ventajas sobre los anestésicos inhalados; por un lado se evitaría la contaminación ambiental, resultado de las frecuentes succiones por el fibrobroncoscopio, y por otro, podrían limitarse los sangrados en la mucosa respiratoria, ya que existe la impresión subjetiva por parte de los autores del trabajo de Sarkiss et $\mathrm{al}^{2}$, de que los halogenados provocan más sangrados locales, debido a una vasodilatación local de los vasos bronquiales y pulmonares. Además las frecuentes succiones por el endoscopio dificultan el asegurar una fracción inhalada determinada del halogenado utilizado.

Otro aspecto controvertido que es común a todos los procedimientos mínimamente invasivos (implantación de válvulas cardíacas transcatéter, toma de biopsias transbronquiales o a través del tracto digestivo, implantación de stents en vía aérea o colangiopancreatografías retrógradas endoscópicas entre otras), y a la cual no es ajena esta técnica, es la conveniencia de realizar los procedimientos en salas con organización orientada a procedimientos médicos (endoscópicos, hemodinámicos, fibrobroncoscópicos) en quirófanos, o en salas híbridas ${ }^{5}$. Probablemente una sala híbrida puede satisfacer las necesidades de los especialistas implicados, aunque suele imponerse una flexibilidad adaptada a la infraestructura y disponibilidad de cada centro.

En resumen, los agentes intravenosos son en principio los más recomendados, y respecto al manejo de la vía aérea, a pesar de que los trabajos de revisión publicados defienden la utilización de anestesia general con relajación neuromuscular y uso de dispositivos supraglóticos o transglóticos, en nuestra experiencia creemos que la técnica admite un manejo algo menos agresivo, con la utilización de una sedación profunda pero preservando

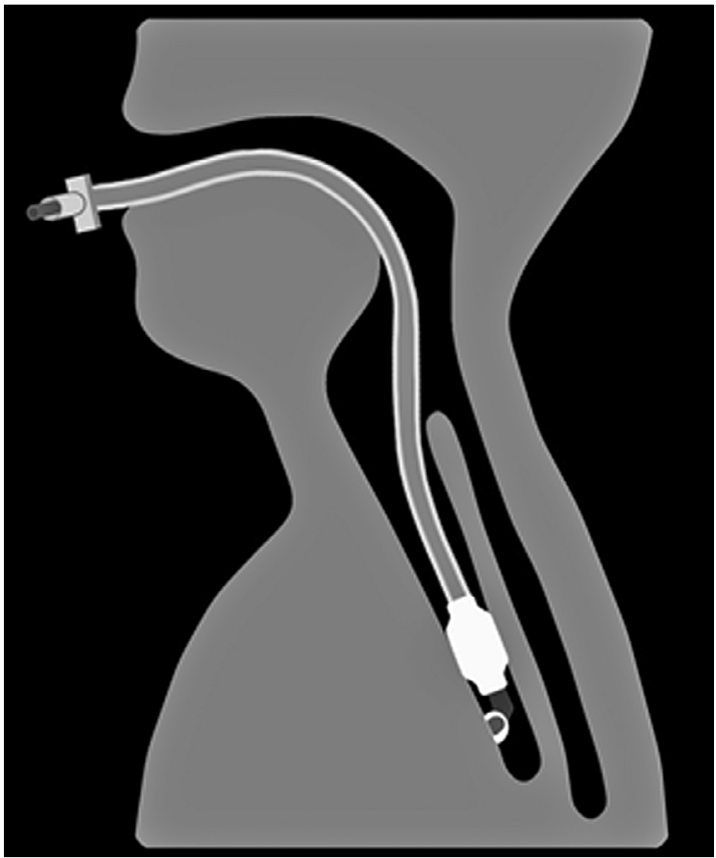

Figura 2. sonda de broncoscopia con ultrasonidos pasando a través de un tubo endotraqueal.

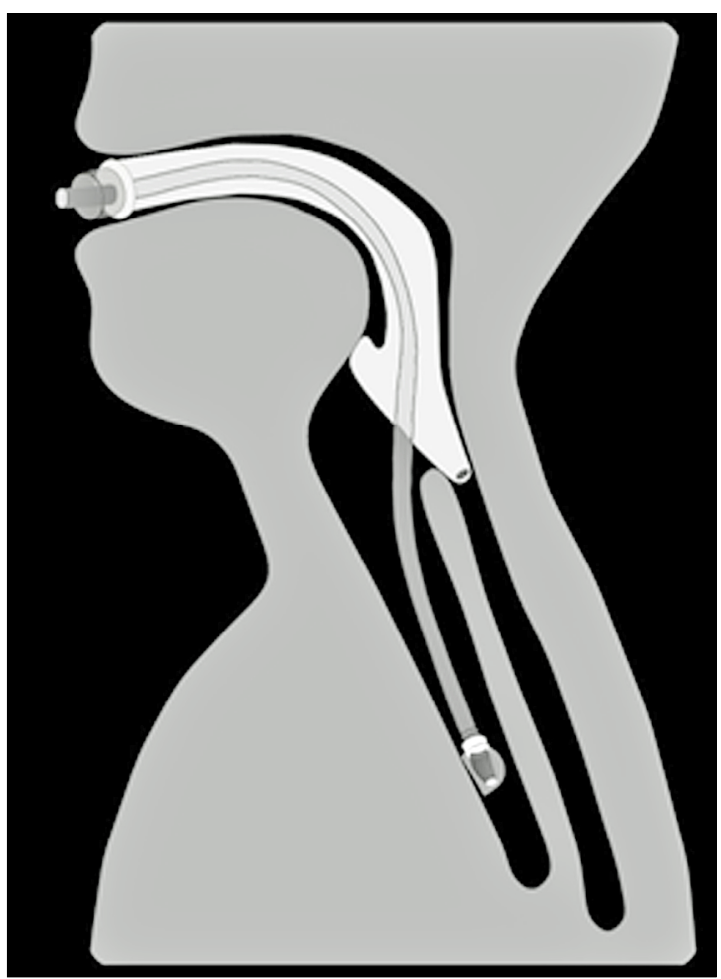

Figura 3. Sonda de broncoscopia equipada con ultrasonidos pasando a través de un dispositivo supraglótico; obsérvese que el acceso permite una alineación más favorable de la sonda para acceder a algunos ganglios paratraqueales. 
la ventilación espontánea y evitando la relajación neuromuscular, lo cual podría redundar en una mejor recuperación del paciente. A pesar de estas recomendaciones sobre el uso de anestesia general, en registros relativos a estos procedimientos que incluyen unos 1.300 casos aproximadamente, hasta un tercio de los casos se han realizado con sedación superficial y aproximadamente un $45 \%$ no precisaron ventilación con presión positiva ${ }^{6}$. Como ocurre en tantas ocasiones durante la actividad diaria del anestesiólogo, debemos elegir entre técnicas anestésicas invasivas, que garantizarían la inmovilidad total del paciente y la ventilación efectiva completa al mismo tiempo que en general aumentan la complejidad, el gasto y en ciertos aspectos la morbilidad (al intervenir directamente sobre la vía aérea) y otras técnicas menos agresivas, como mostramos en esta pequeña serie, que idealmente podrían conseguir la inmovilidad para el éxito del procedimiento pero sin la necesidad de inducir una abolición de la respiración espontánea, sin administrar bloqueantes neuromousculares y evitando la intervención directa sobre la vía aérea; ambas estrategias tienen ventajas e inconvenientes, y creemos que el personal adecuado para tomar estas decisiones, y que tiene a su disposición el arsenal completo de manejo del enfermo con potencial compromiso de la vía aérea y la hemodinámica, debe ser un anestesiólogo. Probablemente habría que individualizar según el tipo de paciente y procedimiento (duración, complejidad) para decidir qué casos son más favorables para realizarse con sedación profunda o con anestesia general ${ }^{7}$.

Declaración de intereses: Todos los autores declaran haber participado en la realización del manuscrito, así como no tener conflicto de intereses. No se ha recibido financiación de ningún tipo.

Agradecimientos: A Andrea López Álvarez, autora de las ilustraciones.

\section{REFERENCIAS}

1. Sánchez de Cosa J, Hernández Hernández J, Jiménez López MF, et al. Normativa SEPAR sobre estadificación del cáncer de pulmón. Archivos de Bronconeumología, 2011, doi:10.1016/j.arbres.2011.06.013.

2. Sarkiss M, Kennedy M, Riedel B, et al. Anesthesia technique for endobronquial ultrasound-guided fine needle aspiration of mediastinal lymph node. Journal of cardiothoracic and vascular anesthesia. 2007; 21: 892-896.
3. Sarkiss M. Anesthesia for bronchoscopy and interventional pulmonology: from moderate sedation to jet ventilation. Current Opinion in Pulmonary Medicine 2011; 17: 274-278.

4. Douadi Y, Bentayeb H, Malinowski S, et al. Anesthésie en échoendoscopie bronchique. Expérience du masque larynge. Revue des Maladies Respiratoires 2010; 27: 37-41.

5. Abdelmalak BA, Gildea TR, Doyle DJ. Anesthesia for bronchoscopy. Current Pharmaceutical Design
2012; 18: 6314-6324.

6. Eapen GA, Shah AM, Lei X, et al. Complications, consequences, and practice patterns of endobronchial ultrasound-guided transbronchial needle aspiration: results of the AQuiRE registry Chest, Nov 2012, ahead of print.

7. Kennedy MP, Shweilat Y, Sarkiss $\mathrm{M}$, et al. Complete mediastinal and hilar lymph node staging of primary lung cancer by endobronchial ultrasound. Moderate sedation or general anesthesia? Chest 2008; 134: 1350-1351.

\footnotetext{
Correspondencia a:

Dra. Alexo López Álvarez

Hospital Meixoeiro de Vigo

(Complejo Hospitalario

Universitario de Vigo). España.

Teléfono: 617542780

lopez.alexo@gmail.com
} 